\title{
Association Analysis under Population Stratification: A Two-Stage Procedure Utilizing Population- and Family-Based Analyses
}

\author{
Hui-Wen Lin ${ }^{a}$ Yi-Hau Chen ${ }^{b}$ \\ ${ }^{a}$ Biostatistics Research and Consulting Center, Taipei Medical University, and ${ }^{b}$ Institute of Statistical Science, \\ Academia Sinica, Taipei, Taiwan, ROC
}

\section{Key Words}

Association study $\cdot$ Case-parents study $\cdot$ Parental

missingness $\cdot$ Population stratification

\begin{abstract}
Objective: The association analysis based on a populationbased case-control study is convenient and powerful, but may be biased under population stratification (PS), namely the study population consists of strata heterogeneous in disease rates and allele frequencies. On the other hand, a family-based (e.g. case-parents) study is robust against the PS bias, but may be less convenient to implement. We propose an association analysis that preserves the full robustness property of the family-based analysis while allowing for borrowing information from a population-based analysis. Methods: A two-stage procedure is proposed. In the first stage, one selects a population-based case-control sample and performs a traditional case-control association analysis. In the second stage, one randomly selects a subset of the firststage cases and recruits their family controls (e.g. parents), and performs a family-based association analysis. An overall two-stage analysis is then performed to utilize information from the two stages. Results: The proposed two-stage analysis achieves higher power than the second-stage family-
\end{abstract}

based analysis by utilizing information in the first-stage population study, while maintaining the full robustness of the family study and hence is still valid under PS. The proposal can also accommodate parental missingness when the caseparents study is used as the second-stage family study. Conclusion: The two-stage analysis facilitates efficient and robust association analysis under PS. Its computation- and cost-effectiveness render it very promising in genome-wide association studies.

Copyright $\odot 2009$ S. Karger AG, Basel

\section{Introduction}

Population-based case-control studies of gene-disease association provide a convenient and powerful approach to identify the genetic variants that modulate susceptibility to complex human diseases. However, such studies are prone to confounding by 'population stratification' (PS) if the study population consists of a few hidden strata across which the allele frequencies and disease rates vary. The confounding bias due to PS results in biased estimates for genotype relative risks and inflated type I error rates in association testing, so that the detected association may be spurious. Such a bias persists even when the

\section{KARGER}

Fax +4161306 1234 E-Mail karger@karger.ch www.karger.com (c) 2009 S. Karger AG, Basel

0001-5652/10/0693-0160\$26.00/0

Accessible online at:

www.karger.com/hhe
Yi-Hau Chen, $\mathrm{PhD}$

Institute of Statistical Science, Academia Sinica

Taipei 11529, Taiwan (ROC)

Tel. +8862 2783 5611, ext. 622, Fax +88622783 1523

E-Mailyhchen@stat.sinica.edu.tw 
sample size is increased. Also, adjustment of PS using traditional matching or stratified methods may not be possible since it is usually hard to identify the hidden population strata.

One strategy for the association analysis under PS is to correct the potential bias for the population-based analysis, with the bias-correction factors assessed from the genotypes of a set of genomic markers for the study subjects. Methods based on this strategy include Genomic Control (GC) [1], Structured Association [2, 3], and Principle Components Analysis (PCA, implemented in the EIGENSTRAT software) $[4,5]$. However, it has been understood that such methods may not eliminate the PS bias completely $[6,7]$. For example, the GC approach may fail when the constant overdispersion assumption is wrong or too few markers are used for correction $[5,8,9]$, and the PCA approach may be biased when markers are insufficient to infer the populations [5] or the multilinear regression assumption is inadequate [6].

Another well-known strategy to protect against the PS bias is to base the association analysis on the 'familybased designs', such as the case-parents and case-sibling designs [10-13]. The family designs use relatives of the cases as 'family controls', so that cases and controls are matched with respect to the ethnic background and hence the PS bias can be naturally eliminated in the consequent association analysis. It is known that [14] family studies can achieve similar statistical power to population studies of comparable size when both parents of the diseased subjects are recruited as family controls (i.e. the case- parents designs). However, a disadvantage for family designs is that it may not be easy to collect genotype data from family controls, such as from parents of the patients with late-onset diseases. Consequently, it is common in genetic association studies to conduct the family-based association study as a smaller-scale, followup validation study for a larger-scale population-based association study. Due to the restriction in sample sizes, the analysis from such a family-based validation study may have limited power to detect the genuine association, even though it has a correct type I error rate. This motivates the efforts to perform association analysis integrating data from both the main population study and the follow-up family study to guarantee a sufficient power; some recent methodologies developed towards this end include, for example, Nagelkerke et al. [15], Epstein et al. [16] and Chen and Lin [17]. Although these proposals can detect the association with data from both the population-based case-control and family studies, they all assume that both the population and the family anal- yses provide valid results, which excludes the case where PS exists.

We propose in this work a two-stage procedure for the association analysis under PS. Specifically, when it is believed that the study population is not homogeneous and contains several strata, we propose to perform the association study in a two-stage manner. In the first stage of the study, one selects a population-based case-control sample and performs a traditional case-control (logistic regression) association analysis. In the second stage, one randomly selects a set of cases from the first-stage cases and recruits family controls (e.g. parents) for the selected cases, and performs a family-based association analysis for the second-stage cases and their recruited family controls. Finally, an overall and refined analysis is performed to combine association analyses from both stages, i.e. the first-stage population-based and the second-stage family-based association analyses.

Compared with existing methods for combining population- and family-based studies [15-17], the two-stage procedure we proposed has the distinct advantages that (1) it does not require assumptions and estimation for the genotype distribution, and (2) it always yields valid results even when there exists PS in the study population. In other words, unlike the other methods available in the literature for combining population- and family-based studies, our new proposal still preserves the full robustness property of the family-based analysis when utilizing the population-based analysis. In contrast to methodologies available for correcting PS bias, such as the GC and PCA methods, the proposed two-stage analysis always yields correct type I error rates without imposing additional assumptions such as constant overdispersion or linearity. Further, as will be discussed later, the proposed two-stage analysis remains valid even when the parental missingness depends on the population stratification and on the parental genotype, provided that the missingness is independent of offspring genotype. Owing to these advantages, the proposed two-stage analysis can enjoy a wider applicability than existing methods.

Although the proposed procedure allows for general family designs such as case-parents and case-sibling studies to be performed at the second stage, in the following discussion we will focus specifically on the setting where a case-parents design is adopted in the second-stage study; namely, the second-stage family-based study is performed on the selected second-stage cases as well as their parents. 


\section{Methods}

Two-Stage Analysis

Suppose that data on genotypes $G$ and/or environmental exposures $E$ are available for a population-based case-control sample of $N_{1}$ cases and $N_{0}$ controls. The assessment of the gene-disease association can be based on the logistic regression model

$$
\log \left\{\frac{\operatorname{Pr}(D=1 \mid G, E)}{\operatorname{Pr}(D=0 \mid G, E)}\right\}=\alpha+\gamma^{T} h(G, E),
$$

where $D$ is an indicator of disease, $h(G, E)$ is a known vector of coded values of $(G, E)$ with $h(\cdot)=0$ for a reference genotype-exposure group, $\alpha$ is an intercept and $\gamma$ is a vector of regression coefficients with respect to genotypes, exposures, and/or genotypeexposure interactions. The parameter $\gamma$ contains population-level association information, whose inference with case-control data can be simply based on the prospective logistic regression analysis [18].

Alternatively, family-level association information can be assessed from the case-parents analysis provided that genotype data $G_{P}$ are available for the parents of the diseased subjects. Specifically, the 'Conditional-on-Parental-Genotypes' (CPG) analysis $[11,19]$ extracts the within-family association information via the relative risks model (with $[G=0, E=0]$ denoting the reference genotype-exposure group)

$$
\log \left\{\frac{\operatorname{Pr}(D=1 \mid G, E)}{\operatorname{Pr}(D=1 \mid G=0, E=0)}\right\}=\beta^{T} h(G, E),
$$

and the corresponding conditional likelihood

$$
\begin{aligned}
\operatorname{Pr}\left(G \mid G_{P}, E, D=1\right) & =\frac{\operatorname{Pr}(D=1 \mid G, E) \operatorname{Pr}\left(G \mid G_{P}\right)}{\sum_{G^{*}} \operatorname{Pr}\left(D=1 \mid G^{*}, E\right) \operatorname{Pr}\left(G^{*} \mid G_{P}\right)} \\
& =\frac{\exp \left\{\beta^{T} h(G, E)\right\} \operatorname{Pr}\left(G \mid G_{P}\right)}{\sum_{G^{*}} \exp \left\{\beta^{T} h\left(G^{*}, E\right)\right\} \operatorname{Pr}\left(G^{*} \mid G_{P}\right)},
\end{aligned}
$$

where $\beta$ is a vector of regression coefficients with respect to genotypes, exposures, and/or genotype-exposure interactions. Note that in (3), $\operatorname{Pr}\left(G \mid G_{P}\right)$ is determined by the Mendelian proportions, and we have assumed the within-family gene-environment independence

$$
\operatorname{Pr}\left(G, E \mid G_{P}\right)=\operatorname{Pr}\left(G \mid G_{P}\right) \times \operatorname{Pr}\left(E \mid G_{P}\right),
$$

namely the genotypes and environmental exposures are independent of each other given parental genotypes, which generally holds even in the presence of PS [19,20].

The population-based case-control study is subject to the confounding bias due to PS, while the case-parents study is robust to this bias. On the other hand, recruiting population (unrelated) controls is usually more convenient than recruiting parents of the diseased subjects. Therefore, it is natural in practice to consider a study design encompassing a larger-scale population-based casecontrol study to gain power, and a smaller-scale case-parents study to ensure unbiasedness. To implement such an idea, we propose a two-stage study design as follows. In the first stage of the study, data on genotypes and/or environmental exposures are collected for $N_{1}$ cases and $N_{0}$ unrelated controls selected from the study population, i.e., a population-based case-control sample. In the second stage, $n_{1}\left(n_{1} \leq N_{1}\right)$ cases are randomly selected from the first-stage case sample, and genotype data are further collected for the parents of the selected second-stage cases. In the following, we refer to the subsample consisting of the selected second-stage cases and their recruited parents as the 'second-stage case-parents sample'. Also, the subsample consisting of the selected second-stage cases and all the first-stage controls is referred to as the 'second-stage case-control sample'.

Suppose that $\bar{\gamma}$ and $\hat{\gamma}$ are estimates of the logistic regression parameter $\gamma$ obtained respectively from the first- and secondstage case-control samples. Let $\hat{\beta}$ be the CPG estimate of the parameter $\beta$ in model (2) obtained from the second-stage case-parents sample. Traditional population- and family-based association analyses would be based respectively on $\bar{\gamma}$ and $\hat{\beta}$. We propose a refined two-stage analysis based on the combined estimate given by

$$
\bar{\beta}=\hat{\beta}-C(\beta, \gamma) V(\gamma)^{-1}(\hat{\gamma}-\bar{\gamma}),
$$

where $C(\beta, \gamma)$ is the covariance matrix between $\hat{\beta}$ and $(\hat{\gamma}-\bar{\gamma})$, and $V(\gamma)$ is the variance matrix of $(\hat{\gamma}-\bar{\gamma})$; these variance-covariance matrices can be estimated using simple sandwich estimates given in Appendix.

The combined estimate $\bar{\beta}$ has a similar form to the estimate in Chen and Chen [21], which is originally proposed for two-stage designs where the second-stage sample is a random subset of the first-stage sample and has been applied for combining the familybased analysis with the case-only analysis for detecting gene-environment interaction [22]. Observe that, like the case-parents estimate $\hat{\beta}$, the combined $\bar{\beta}$ is always asymptotically unbiased even in the presence of PS, since, recalling the fact that the second-stage cases are a random subset of the first-stage cases and the classical result in Prentice and Pyke [18], the two estimates for $\gamma$, i.e. the first- and second-stage case-control (logistic regression) estimates $\hat{\gamma}$ and $\bar{\gamma}$, would have the same asymptotic limit and hence the second term in the right side of (4) is asymptotically zero, no matter the case-control estimates are unbiased or not. In other words, the validity of the proposed two-stage analysis does not require more assumptions than those required for the validity of the case-parents analysis. This distinct advantage is not shared by other methods combining population- and familybased analyses [e.g., 15-17], all of which assume a homogeneous population and become invalid in the presence of PS.

The variance matrix of $\bar{\beta}$ is given by

$$
\operatorname{Var}(\bar{\beta})=\operatorname{Var}(\hat{\beta})-C(\beta, \gamma) V(\gamma)^{-1} C(\beta, \gamma)^{T} .
$$

Therefore, we see that the combined estimate $\bar{\beta}$, further utilizing information from the first-stage case-control sample, is more efficient (with smaller asymptotic variance) than the secondstage case-parents estimate $\hat{\beta}$. When the second-stage sampling fraction $n_{1} / N_{1}$ is increased towards 1 , the combined estimate $\beta$ would come close to the corresponding case-parents estimate $\hat{\beta}$. Note that when $n_{1}=N_{1}=N_{0}$, the results in Laird and Lange ([14], fig. 1) reveal that the case-parents estimate $\hat{\beta}$ would yield similar testing power to the population-based case-control estimate $\bar{\gamma}$ in the absence of PS.

The gene-disease association test combining information from both the population-based case-control and the case-parents studies can then be performed by the Wald test based on suit- 


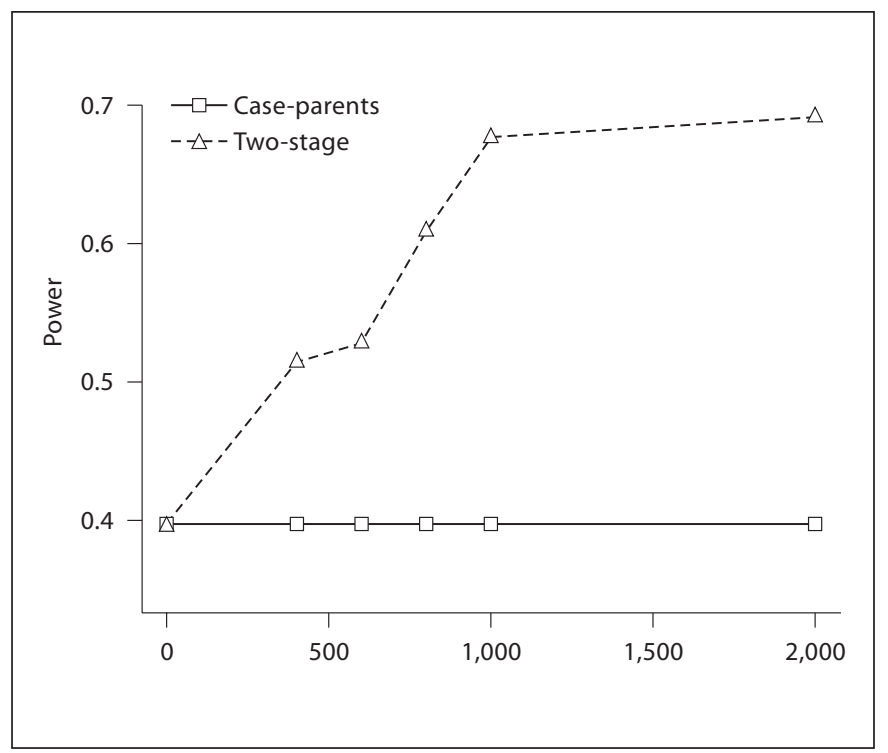

Fig. 1. Power of the proposed two-stage analysis for detecting genotype effect at $5 \%$ significance level. The number of families used in the second stage is 200 , while the number of the first-stage cases (controls) increases from 400 to 2,000. Data are simulated under the setting with $q=0.7$ and $k=0.1$, and recessive genetic model with $\beta_{g}=0.4$.

able components in $\bar{\beta}$ and $\operatorname{Var}(\bar{\beta})$, and standard $\chi^{2}$ distributions with suitable degrees of freedom. According to the above discussion, the association test based on the combined estimate $\bar{\beta}$ would be more powerful than the test based only on the second-stage case-parents estimate, and also more robust to the PS bias than the test based on the first-stage case-control estimate. We will examine this property in simulation studies.

\section{Parental Missingness and Nonrandom Selection of the Second-Stage Sample}

The case-parents study often encounters the problem of parental missingness, i.e. one or both parents are missing for some families studied. This is especially the case when a late-onset disease is studied. A nice feature enjoyed by the case-parents analysis is that, the case-parents analysis based on 'complete trios' (families with both parents available) is still valid if the parental missingness is independent of offspring's genotypes $G$ given the parental genotypes $G_{P}$, offspring's disease status $D=1$ and exposures $E$ (if available), and subpopulation membership $Z$. Symbolically, let $R$ denote the parental missingness pattern (the missing indicators for the two parents in a family). The missingness mechanism required for the complete-trio analysis to be valid satisfies the following relationship

$$
\operatorname{Pr}\left(R \mid G_{P}, G, D=1, Z\right)=\operatorname{Pr}\left(R \mid G_{P}, D=1, Z\right)
$$

[22-24]. Note that such a missing mechanism allows the parental missingness to depend on the missing parental genotype themselves, which is a type of 'informative' missingness that is more

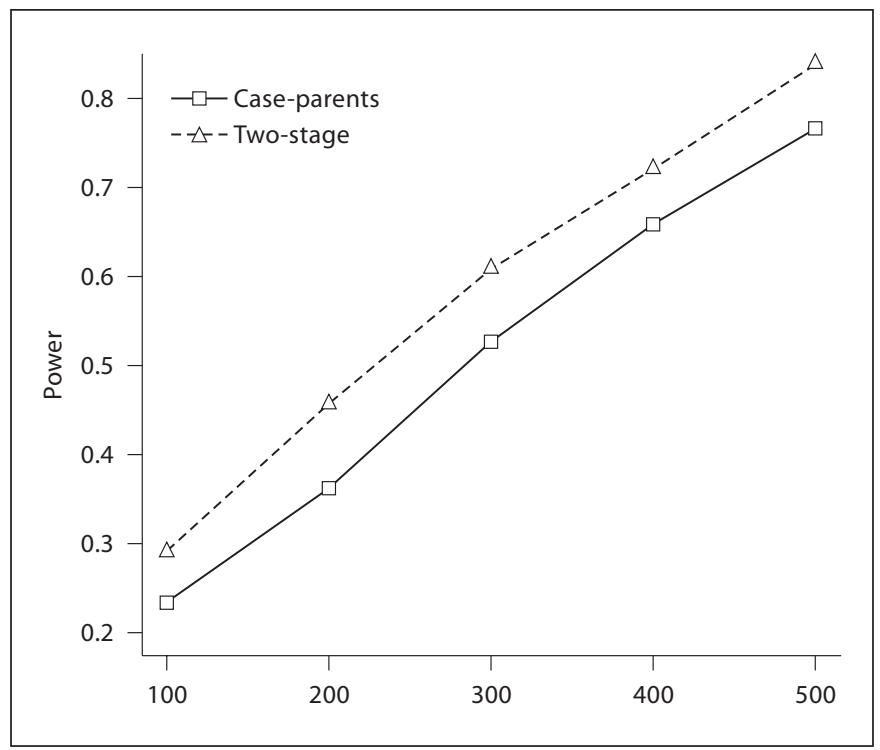

Fig. 2. Power of the proposed two-stage analysis for detecting genotype effect at $5 \%$ significance level. The number of first-stage cases (controls) is 1,000, while the number of the second-stage families increases from 100 to 500. Data are simulated under the setting with $q=0.7$ and $k=0.1$, and recessive genetic model with $\beta_{g}=0.4$.

general than the 'missing-at-random' missingness assumed by usual methods dealing with missing data [25-28]. Besides, the mechanism (5) allows the parental missingness to depend on PS.

From a more general perspective, we can view the process for 'parental missingness' as the process for 'selection/participation' of the parents of the cases for the second-stage study. Consequently, our proposal in fact allows for non-random selection/participation of the case-parents trios for the second-stage study, as long as the relation (5) holds; that is, given other factors, the selection/ participation mechanism depends on the genotypes of the trio only through the genotypes of the parents.

\section{Recovering Missing Parental Information}

Even though the case-parents analysis based on complete trios ensures unbiasedness under the parental missingness mechanism (5), the testing power may be dramatically reduced with a substantial amount of missingness. To recover the missing information, Chen [24] and Chen et al. [22] have extended the traditional case-parents analysis to accommodate incomplete trios (families with one or both parents missing), which is based on the conditional likelihood of the offspring's genotypes given the genotypes of the observed parents (if any), the offspring's disease status and exposures (if available), and the number of observed parents; see Chen [24] (without environmental covariates) and Chen et al. ([22], Appendix) (with environmental covariates) for details of the conditional likelihood. This approach further exploits incomplete trios but still maintains the full robustness property of the traditional case-parents analysis, in the sense that it does not require specifying and estimating the genotype distributions and 
the missingness probabilities, and is valid even under PS, as long as the parental missingness mechanism satisfies (5). We suggest applying this conditional likelihood approach to obtain the second-stage case-parents estimate $\hat{\beta}$ and hence the combined estimate $\bar{\beta}$ in the proposed two-stage analysis when parental missingness occurs in the second-stage case-parents study.

\section{Simulations and Results}

A series of simulation experiments have been performed to examine the performance of the proposed twostage association analysis under PS, based on models without or with the gene-environment interaction. We assume that the study population comprises two subpopulations (denoted respectively by $Z=1$ and $Z=2$ ), and the disease gene locus is diallelic. The overall disease rates in the two subpopulations are respectively 1 and $5 \%$, and the frequencies of the disease-causing allele in the two subpopulations are $p_{1}=0.3$ and $p_{2}=0.3+k$, where $k=0.05$ or 0.1. Genotypes for offsprings are generated according to Hardy-Weinberg equilibrium. The models used for generating offspring's disease status will be detailed later. When analyzing the data, we use the disease model same as the one generating the data (the true model). The firststage case-control sample is obtained by collecting $N$ diseased (case) and $N$ non-diseased (control) offsprings. The selection probabilities in the first and second subpopulations for the cases are respectively $q$ and $1-q$, and the corresponding probabilities for the controls are $1-q$ and $q$, with $q=0.5$ or 0.7 . Note that the quantify $q$ reflects the degree of differential selection in cases and controls, with $q=0.5$ meaning non-differential selection. A larger degree of differential selection would lead to a stronger PS level $[7,29]$. By varying the values for $q$ and $k$ (the allelefrequency difference between strata), we consider different levels of PS, with larger values of $|q-0.5|$ and $k$ meaning greater levels of PS. The second-stage case-parents sample is obtained by randomly selecting $n$ cases from the first-stage case sample and then recruiting the parents of the $n$ selected cases. Evaluations of type I error rate/power are based on 1,000 simulated samples.

\section{Model without Gene-Environment Interaction}

In the first simulation study, the offspring's disease status is generated by the model

$$
\operatorname{Pr}(D=1 \mid G, Z)=C_{Z} \exp \left\{\beta_{g} h(G)\right\},
$$

where $h(G)$ denotes offspring's genotypic code at the disease locus according to the chosen penetrance model (dominant, recessive, or multiplicative), the subpopula- tion-dependent constant $C_{Z}$ is specified to yield the desired overall disease rates (1\% when $Z=1$ and $5 \%$ when $Z=2$ ), and $\beta_{g}=0.3 ; 0.4$ or 0.6 for different degrees of association. In terms of relative penetrance or genotypic risk ratios $f_{k} \equiv \operatorname{Pr}(D=1 \mid G=k, Z) / \operatorname{Pr}(D=1 \mid G=0, Z)$, $k=1,2$, the considered disease models have $f_{2}=f_{1}=1.5$ or 1.8 for the dominant model, $f_{2}=1.5$ or 1.8 for the recessive model $\left(f_{1}=1\right)$, and $f_{2}=1.7, f_{1}=1.3$ or $f_{2}=2.3, f_{1}=$ 1.5 for the multiplicative model. The proposed two-stage association analysis is performed with genotype data from the first-stage case-control sample, as well as the parental genotype data for the second-stage cases randomly selected from the first-stage case sample. The sampling fraction $n / N$ for the second-stage case-parents study is $100 / 800(0.125), 200 / 800(0.25)$ or $300 / 900(0.33)$.

In this study we also assume that a panel of markers unlinked to the disease locus are available for the full first-stage case-control sample, so that we can apply the GC and PCA (EIGENSTRAT) methods to correct the case-control (logistic regression) analysis based on the first-stage sample. The allele frequencies of the unlinked loci in each subpopulation are randomly generated from the uniform distribution $U\left(p_{Z}-0.05, p_{Z}+0.05\right), Z=1,2$, where $p_{Z}$ is the frequency of the disease-causing allele in subpopulation $Z\left(p_{1}=0.3\right.$ and $p_{2}=0.3+k$ as mentioned above).

Table 1 shows type I error rates for the various methods under the null hypothesis that no disease locus is in linkage disequilibrium with the marker being tested for association. The type-I error rates for the populationbased case-control analysis are seriously inflated in the settings considered, while those for the case-parents analysis are correct. The use of the GC method with 60 unlinked markers, though conservative when there exist substantial degrees of PS (e.g. when $q=0.5$ and $k=0.1$ ), successfully removes the bias of the population-based case-control analysis, while the PCA analysis with 60 unlinked markers exhibits inflated type I error rates, and this phenomenon persists even when the number of unlinked markers is increased to 500 (data not shown). The PCA analysis turns out to be valid (i.e. has correct type I error rates) when the number of markers is increased to 1,000 . On the other hand, the proposed two-stage analysis, without the need of genotyping any additional markers, maintains the robustness property of family-based analysis and provides correct type-I error rates.

We also assess the type I error rates for the method proposed by Epstein et al. [16], which, like our proposal, combines data from a population case-control sample and a set of parental controls. We find that, in the PS set- 
Table 1. Type I error rates of the various association tests for testing $\beta_{g}=0$ at $5 \%$ significance level, including the tests based on the first-stage case-control estimate $\bar{\gamma}$, the second-stage case-parents estimate $\hat{\beta}$, the proposed two-stage estimate $\bar{\beta}$, the genomic control method GC with 60 markers, and the principle components analysis PCA with 60 and 1,000 markers

\begin{tabular}{|c|c|c|c|c|c|c|c|}
\hline \multirow[t]{2}{*}{ Setting } & & \multicolumn{6}{|c|}{ Type I error rates of test based on } \\
\hline & & $\bar{\gamma}$ & $\hat{\beta}$ & $\bar{\beta}$ & GC(60) & $\operatorname{PCA}(60)$ & $\operatorname{PCA}(1,000)$ \\
\hline \multicolumn{8}{|c|}{$n / N=100 / 800$} \\
\hline no PS & & 0.052 & 0.048 & 0.049 & 0.046 & 0.045 & 0.047 \\
\hline \multirow[t]{2}{*}{$q=0.5$} & $k=0.05$ & 0.172 & 0.054 & 0.058 & 0.033 & 0.118 & 0.059 \\
\hline & $k=0.1$ & 0.531 & 0.051 & 0.041 & 0.004 & 0.135 & 0.045 \\
\hline \multirow[t]{2}{*}{$q=0.7$} & $k=0.05$ & 0.444 & 0.039 & 0.038 & 0.013 & 0.368 & 0.053 \\
\hline & $k=0.1$ & 0.969 & 0.053 & 0.044 & 0 & 0.434 & 0.057 \\
\hline \multicolumn{8}{|c|}{$n / N=200 / 800$} \\
\hline no PS & & 0.051 & 0.051 & 0.052 & 0.055 & 0.045 & 0.048 \\
\hline \multirow[t]{2}{*}{$q=0.5$} & $k=0.05$ & 0.158 & 0.048 & 0.052 & 0.042 & 0.116 & 0.060 \\
\hline & $k=0.1$ & 0.538 & 0.046 & 0.032 & 0 & 0.138 & 0.043 \\
\hline \multirow[t]{2}{*}{$q=0.7$} & $k=0.05$ & 0.447 & 0.056 & 0.051 & 0.008 & 0.370 & 0.056 \\
\hline & $k=0.1$ & 0.954 & 0.053 & 0.056 & 0 & 0.438 & 0.057 \\
\hline \multicolumn{8}{|c|}{$n / N=300 / 900$} \\
\hline no PS & & 0.046 & 0.046 & 0.056 & 0.060 & 0.042 & 0.047 \\
\hline \multirow{2}{*}{$q=0.5$} & $k=0.05$ & 0.171 & 0.043 & 0.051 & 0.041 & 0.158 & 0.058 \\
\hline & $k=0.1$ & 0.577 & 0.046 & 0.053 & 0.010 & 0.174 & 0.054 \\
\hline \multirow[t]{2}{*}{$q=0.7$} & $k=0.05$ & 0.528 & 0.045 & 0.052 & 0.011 & 0.410 & 0.060 \\
\hline & $k=0.1$ & 0.975 & 0.046 & 0.058 & 0 & 0.485 & 0.056 \\
\hline
\end{tabular}

tings considered here, the Epstein et al. method is invalid. For example, in the setting of table 1 with $n / N=200 / 800$, $q=0.5$ and $k=0.05$, the Epstein et al. method has a seriously inflated type I error rate of 0.985 . This result confirms the fact that the Epstein et al. method may be biased under PS [16, p 594].

Results of the test power shown in table 2 reveal that the proposed two-stage analysis effectively improves the power of the second-stage case-parents analysis; for example, when $25 \%$ cases are selected for the second-stage case-parents study, the power improvement ranges from 15 to $60 \%$, with greater improvement obtained under a weaker recessive/dominant genetic effect. Compared with the results by using 60 additional unlinked markers and applying the GC approach to the full first-stage case-control sample, the two-stage analysis is less powerful under moderate degrees of PS, but becomes equivalently or more powerful when there exist substantial degrees of PS ( $q=$ 0.7 and $k=0.1$ ). The loss of power for the GC approach under substantial PS has also been observed in other studies $[7,8]$. Increasing the number of markers from 60 to 1,000 does not affect the power of the GC approach remarkably (results not shown). In table 2 we also show the power of the PCA analysis with 1,000 unlinked markers, which is higher than that of the proposed and the GC methods in almost all the settings. Recall that the PCA analysis fails to provide correct type I error rates when a smaller number of unlinked markers such as 60 or 500 is used, implying that the power advantage of a valid PCA analysis over the two-stage and GC approaches may be achieved at the expense of higher genotyping costs.

To see how the two-stage analysis can utilize information from the first-stage population study to enhance power, we use the above setups with $q=0.7, k=0.1$ and $\beta_{g}=$ 0.4 (recessive model), and fix the number of families used in the second stage at $n=200$ but gradually increases the number $N$ of the first-stage cases (and also controls) from 400 to 2,000. The power curve in figure 1 illustrates that the proposed two-stage analysis can adaptively exploit the increased population-based information in the first stage sample to enhance its power to detect association.

On the other hand, figure 2 shows the power curves when we fix the number $N$ of the first-stage cases (and also controls) at $N=1,000$, but varies the number $n$ of the second-stage families from 100 to 500 . It is seen that the proposed two-stage analysis has satisfactory power when 
Table 2. Power of the various association tests for testing $\beta_{g}=0$ at $5 \%$ significance level, including the tests based on the second-stage case-parents estimate $\hat{\beta}$, the proposed two-stage estimate $\hat{\beta}$, the genomic control method GC with 60 markers, and the principle components analysis PCA with 1,000 markers

\begin{tabular}{|c|c|c|c|c|c|c|c|c|}
\hline \multirow[t]{2}{*}{ Setting } & & \multirow[t]{2}{*}{ Method } & \multicolumn{2}{|c|}{ Multiplicative model, $\beta_{g}$} & \multicolumn{2}{|c|}{ Recessive model, $\beta_{g}$} & \multicolumn{2}{|c|}{ Dominant model, $\beta_{g}$} \\
\hline & & & 0.3 & 0.4 & 0.4 & 0.6 & 0.4 & 0.6 \\
\hline \multicolumn{9}{|c|}{$n / N=200 / 800$} \\
\hline \multirow{8}{*}{$q=0.5$} & $k=0.05$ & $\hat{\beta}$ & 0.534 & 0.794 & 0.376 & 0.672 & 0.522 & 0.864 \\
\hline & & $\bar{\beta}$ & 0.724 & 0.930 & 0.574 & 0.894 & 0.794 & 0.992 \\
\hline & & $\mathrm{GC}(60)$ & 0.999 & 0.999 & 0.844 & 0.930 & 0.924 & 0.999 \\
\hline & & $\operatorname{PCA}(1,000)$ & 0.995 & 1 & 0.845 & 0.985 & 0.965 & 1 \\
\hline & $k=0.1$ & $\hat{\beta}$ & 0.562 & 0.778 & 0.394 & 0.750 & 0.500 & 0.802 \\
\hline & & $\bar{\beta}$ & 0.722 & 0.924 & 0.614 & 0.928 & 0.740 & 0.960 \\
\hline & & $\mathrm{GC}(60)$ & 0.933 & 0.999 & 0.812 & 0.954 & 0.922 & 1 \\
\hline & & $\operatorname{PCA}(1,000)$ & 0.955 & 1 & 0.805 & 0.990 & 0.960 & 1 \\
\hline \multirow{9}{*}{$q=0.7$} & $k=0.05$ & $\hat{\beta}$ & 0.516 & 0.762 & 0.324 & 0.680 & 0.530 & 0.836 \\
\hline & & $\bar{\beta}$ & 0.734 & 0.924 & 0.518 & 0.886 & 0.778 & 0.986 \\
\hline & & $\mathrm{GC}(60)$ & 0.956 & 0.999 & 0.812 & 0.994 & 0.858 & 0.995 \\
\hline & & $\operatorname{PCA}(1,000)$ & 0.970 & 1 & 0.830 & 0.990 & 0.940 & 1 \\
\hline & $k=0.1$ & $\bar{\gamma}_{g}$ & 1 & 1 & 1 & 1 & 1 & 1 \\
\hline & & $\hat{\beta}$ & 0.528 & 0.806 & 0.410 & 0.734 & 0.478 & 0.800 \\
\hline & & $\bar{\beta}$ & 0.722 & 0.928 & 0.602 & 0.932 & 0.796 & 0.968 \\
\hline & & $\mathrm{GC}(60)$ & 0.588 & 0.929 & 0.518 & 0.958 & 0.524 & 0.970 \\
\hline & & $\operatorname{PCA}(1,000)$ & 0.952 & 0.996 & 0.720 & 0.975 & 0.880 & 0.998 \\
\hline \multicolumn{9}{|c|}{$n / N=300 / 900$} \\
\hline \multirow{8}{*}{$q=0.5$} & $k=0.05$ & $\hat{\beta}$ & 0.702 & 0.926 & 0.442 & 0.852 & 0.698 & 0.958 \\
\hline & & $\bar{\beta}$ & 0.858 & 0.982 & 0.697 & 0.962 & 0.906 & 0.998 \\
\hline & & $\mathrm{GC}(60)$ & 0.996 & 1 & 0.888 & 0.992 & 0.958 & 1 \\
\hline & & $\operatorname{PCA}(1,000)$ & 0.995 & 1 & 0.845 & 0.990 & 0.960 & 1 \\
\hline & $k=0.1$ & $\hat{\beta}$ & 0.714 & 0.928 & 0.552 & 0.884 & 0.646 & 0.960 \\
\hline & & $\bar{\beta}$ & 0.886 & 0.986 & 0.752 & 0.990 & 0.864 & 0.998 \\
\hline & & $\mathrm{GC}(60)$ & 0.952 & 1 & 0.868 & 0.994 & 0.956 & 0.998 \\
\hline & & $\operatorname{PCA}(1,000)$ & 0.995 & 1 & 0.875 & 0.995 & 0.960 & 1 \\
\hline \multirow[t]{8}{*}{$q=0.7$} & $k=0.05$ & $\hat{\beta}$ & 0.712 & 0.914 & 0.450 & 0.848 & 0.692 & 0.964 \\
\hline & & $\bar{\beta}$ & 0.858 & 0.980 & 0.688 & 0.966 & 0.890 & 1 \\
\hline & & $\mathrm{GC}(60)$ & 0.954 & 1 & 0.808 & 0.990 & 0.948 & 1 \\
\hline & & $\operatorname{PCA}(1,000)$ & 0.995 & 1 & 0.850 & 0.990 & 0.960 & 1 \\
\hline & $k=0.1$ & $\hat{\beta}$ & 0.708 & 0.944 & 0.548 & 0.888 & 0.652 & 0.960 \\
\hline & & $\bar{\beta}$ & 0.876 & 0.988 & 0.770 & 0.982 & 0.876 & 0.996 \\
\hline & & $\mathrm{GC}(60)$ & 0.536 & 0.948 & 0.524 & 0.954 & 0.526 & 0.974 \\
\hline & & $\operatorname{PCA}(1,000)$ & 0.960 & 0.998 & 0.833 & 0.993 & 0.938 & 0.995 \\
\hline
\end{tabular}

the number of the second-stage families is greater than 300 , i.e., the sampling fraction $n / N$ is greater than 0.3 .

\section{Model with Gene-Environment Interaction}

In the second simulation study, the disease status for the offspring in each family is generated according to the model

$$
\operatorname{Pr}(D=1 \mid G, E, Z)=C_{Z} \exp \left\{\beta_{g} h(G)+\beta_{e} E+\beta_{g e} h(G) E\right\},
$$

where $E$ represents a binary environmental exposure with frequencies (independent of genotypes) 0.2 and 0.4 respectively in the first and second subpopulations, $\beta_{e}=$ $0.3,\left(\beta_{g}, \beta_{g e}\right)=(0,0) ;(0.3,0.3)$ or $(0.4,0.4)$ for different degrees of association, and the subpopulation-specific constant $C_{Z}$ is chosen to yield the desired overall disease rates ( $1 \%$ when $Z=1$ and $5 \%$ when $Z=2$ ). The level of PS is specified by $k=0.1$ and $q=0.5$ or 0.7 . The proposed two-stage association analysis is performed with geno- 
Table 3. Type I error rate/power of the various association tests for testing $\beta_{g}=\beta_{g e}=0$ at $5 \%$ significance level, including the tests based on the first-stage case-control estimate $\bar{\gamma}$, the second-stage case-parents estimate $\tilde{\beta}$, using complete trios, the second-stage case-parents estimate $\hat{\beta}$ using complete and incomplete trios, and the two-stage estimate $\bar{\beta}$

\begin{tabular}{|c|c|c|c|c|c|c|c|c|}
\hline & \multirow[b]{2}{*}{ Method } & \multicolumn{3}{|c|}{ Multiplicative model, $\left(\beta_{g}, \beta_{g e}\right)$} & \multicolumn{2}{|c|}{ Recessive model, $\left(\beta_{g}, \beta_{g e}\right)$} & \multicolumn{2}{|c|}{ Dominant model, $\left(\beta_{g}, \beta_{g e}\right)$} \\
\hline & & $(0,0)$ & $(0.3,0.3)$ & $(0.4,0.4)$ & $(0.3,0.3)$ & $(0.4,0.4)$ & $(0.3,0.3)$ & $(0.4,0.4)$ \\
\hline \multirow[t]{4}{*}{$q=0.5$} & $\bar{\gamma}$ & 0.431 & 1 & 1 & 1 & 1 & 1 & 1 \\
\hline & $\tilde{\beta}$ & 0.040 & 0.796 & 0.974 & 0.404 & 0.602 & 0.478 & 0.686 \\
\hline & $\hat{\beta}$ & 0.052 & 0.798 & 0.974 & 0.440 & 0.706 & 0.524 & 0.760 \\
\hline & $\bar{\beta}$ & 0.051 & 0.848 & 0.990 & 0.497 & 0.740 & 0.622 & 0.840 \\
\hline \multirow[t]{4}{*}{$q=0.7$} & $\bar{\gamma}$ & 0.918 & 1 & 1 & 1 & 1 & 1 & 1 \\
\hline & $\tilde{\beta}$, & 0.034 & 0.506 & 0.832 & 0.416 & 0.658 & 0.520 & 0.794 \\
\hline & $\hat{\beta}$ & 0.042 & 0.520 & 0.838 & 0.482 & 0.704 & 0.568 & 0.812 \\
\hline & $\bar{\beta}$ & 0.042 & 0.584 & 0.876 & 0.536 & 0.750 & 0.652 & 0.896 \\
\hline
\end{tabular}

type and exposure data from the first-stage case-control sample with 900 cases and 900 controls, as well as the parental genotype data for 300 second-stage cases randomly selected from the first-stage case sample. Note that data on the parental environmental covariate are not required for the case-parents and the proposed two-stage analyses.

In this study, we allow the parents for the cases selected at stage two to be missing. The parental missing probability depends on the parental genotype and subpopulation: the missing probability is 0.1 (if $Z=1$ ) or 0.2 (if $Z=$ 2) for parents with 0 or 1 disease-causing allele, and the missing probability is 0.3 (if $Z=1$ ) or 0.4 (if $Z=2$ ) for parents with 2 disease-causing alleles. On average, such missing mechanism leads to $65 \%$ complete trios (families with both parents available), $30 \%$ dyads (families with one parent available), and 5\% monads (families with no parent available). The proposed conditional likelihood approach utilizing both complete and incomplete trios is applied to obtain the second-stage case-parents estimate $\hat{\beta}$ and hence the combined estimate $\bar{\beta}$. As a comparison, we also obtain the estimate $\tilde{\beta}$ based only on complete trios by applying traditional case-parents analysis.

Listed in table 3 are the type I error rate/power of the association tests for testing the null hypothesis of $\beta_{g}=$ $\beta_{g e}=0$. We consider this type of tests because, according to Kraft et al. [30], the joint test of the marginal genetic effect and the gene-environment interaction may be more powerful than the test based only on the marginal genetic effect for detecting gene-disease association. The proposed two-stage estimates $\hat{\beta}$ yield type-I error rates close to the expected value of $5 \%$, as do the case-parents estimates $\tilde{\beta}$ using complete trios and $\hat{\beta}$ using both com- plete and incomplete trios. The first-stage case-control (logistic regression) analysis, however, produces exceedingly high type-I error rates under the levels of PS considered. Results of test power show that the case-parents analysis utilizing both complete and incomplete trios is more powerful compared to the case-parents analysis based only on complete trios, and further power can be gained by the use of the two-stage analysis utilizing complete and incomplete trios as well as the population-based case-control sample.

\section{Discussion}

Teng and Risch [31] previously suggested the possibility of using 'the same group of affecteds in a two-stage process - that is, first comparing them to unrelated controls to increase power to identify candidate loci and then comparing these same affected individuals to familybased controls (parents or unaffected sibs) for robustness'. They further commented that the two tests in this approach will be correlated 'so the threshold for significance for the second test needs to be constructed taking this correlation account'. In this work, we use a similar two-stage strategy to achieve both efficiency and robustness in association studies, but our strategy avoids the difficulty of needing to adjust the significance level of a second test. Instead of using the same group of affecteds in a sequential two-stage manner as suggested by Teng and Risch, we suggested a cost-effective two-stage design, where a larger-scale population-based case-control study is performed at the first stage and a smaller-scale caseparents study is performed at the second stage of the 
study. The estimators obtained respectively from the two stages are combined via the regression estimator proposed in Chen and Chen [21], which automatically accounts for the correlation between estimates obtained in the two stages, and the corresponding Wald test has a standard $\chi^{2}$ distribution under the null hypothesis. No special treatment for finding the significance threshold is required in our proposal.

The new proposal possesses some nice features. It preserves the full robustness of family designs against the PS bias, while in the mean time borrowing information from population designs to enhance power. In particular, 'informative' parental missingness which depends on the population stratification and parental genotype will not invalidate the proposed method, as long as the parental missingness is independent of the offspring genotype. The new proposal can further utilize information from families with only one parent available by applying the conditional likelihood approach of Chen [24] and Chen et al. [22].

Our simulation results reveal that the proposed twostage analysis remains unbiased even when the first-stage case-control analysis is seriously biased, and achieves substantial power gains over the second-stage case-parents analysis. Compared with the genomic control approach, the two-stage analysis may be less powerful under modest PS but may be more powerful under substantial PS. Our proposal is less powerful than the PCA analysis, but the validity of the PCA analysis relies on the additional use of sufficient markers for separating the populations. $\mathrm{R}$ codes implementing the proposed method are freely available on http://www.stat.sinica.edu.tw/ yhchen/download.htm.

Although the PS problem may be minimized in association studies where the cases and controls are well matched for ethnic background, there still exist situations where the degrees of PS may not be safely eliminated by existing tools such as the GC and PCA approaches. In populations with extensive admixture such as African-descent populations resident in Europe and North America, methods correcting for 'average' genome-wide levels of ethnic admixture, such as the GC and PCA methods, may yield spurious associations [32]. Furthermore, consideration of increasing power has inspired the collaborative studies combining a number of separately designed case-control studies, which are likely to encounter remarkable PS due to heterogeneity in individuals belonging to separate populations with structurally different allele frequency patterns [33]. According to our simulation results, our proposal would
Table 4. Power of the association tests based on the first-stage case-control estimate $\bar{\gamma}$ and the two-stage estimate $\bar{\beta}$ for testing $H_{0}: \beta=0$ at $5 \%$ significance level, under the setting without population stratification

\begin{tabular}{|c|c|c|c|c|c|c|}
\hline & \multicolumn{2}{|c|}{$\begin{array}{l}\text { Multiplicative } \\
\text { model, } \beta_{\mathrm{g}}\end{array}$} & \multicolumn{2}{|c|}{$\begin{array}{l}\text { Recessive } \\
\text { model, } \beta_{\mathrm{g}}\end{array}$} & \multicolumn{2}{|c|}{$\begin{array}{l}\text { Dominant } \\
\text { model, } \beta_{\mathrm{g}}\end{array}$} \\
\hline & 0.2 & 0.3 & 0.4 & 0.6 & 0.3 & 0.4 \\
\hline \multicolumn{7}{|c|}{$n / N=200 / 800$} \\
\hline $\bar{\gamma}$ & 0.804 & 0.988 & 0.748 & 0.990 & 0.886 & 0.994 \\
\hline $\bar{\beta}$ & 0.380 & 0.728 & 0.478 & 0.840 & 0.578 & 0.790 \\
\hline \multicolumn{7}{|c|}{$n / N=400 / 800$} \\
\hline $\bar{\gamma}$ & 0.804 & 0.988 & 0.748 & 0.990 & 0.886 & 0.994 \\
\hline $\bar{\beta}$ & 0.572 & 0.936 & 0.696 & 0.986 & 0.760 & 0.954 \\
\hline \multicolumn{7}{|c|}{$n / N=800 / 800$} \\
\hline $\bar{\gamma}$ & 0.804 & 0.988 & 0.748 & 0.990 & 0.886 & 0.994 \\
\hline $\bar{\beta}$ & 0.742 & 0.982 & 0.796 & 0.986 & 0.898 & 0.988 \\
\hline
\end{tabular}

still provide valid analysis and be clearly preferred over alternative methods in this type of studies. Another interesting application of our proposal is that it can also be used in a single-stage manner for studies where parental DNA is available at the beginning of the study for a subset of disease cases.

The robustness and efficiency properties mentioned above make the proposed two-stage analysis very useful for association analysis in the presence of PS. However, when the study population is rather homogeneous such that a population-based case-control analysis is itself valid and efficient, the proposed two-stage design would become less useful since the combined estimate $\bar{\beta}$ would be less efficient than the case-control estimate $\bar{\gamma}$. This fact is illustrated with a simulation study performed under the setting of a homogeneous population, where data are all simulated from the first subpopulation considered in table 2. Results in table 4 show that, when there exists no PS, the proposed two-stage analysis may be remarkably less powerful than the first-stage case-control analysis unless the second-stage sampling fraction $n_{1} / N_{1}$ is large.

In summary, we have developed a systematic twostage procedure that facilitates powerful and robust association analysis in the presence of population stratification. The new proposal is both computation- and costeffective, hence it would serve as a very promising tool in genome-wide association studies; examination on such an extension will be reported in a separate work. 


\section{Appendix: Estimators for the Variance-Covariance Matrices Used in (4)}

At the first stage of the two-stage design proposed, we collect genotype and/or exposure data $\left\{D_{i}, G_{i}, E_{i}, i \in \mathcal{S}_{1}\right\}$ for a sample of cases and unrelated controls, where $\mathcal{S}_{1}$ is the index set of the first-stage sample. A case-control analysis is performed by fitting a logistic regression model (1) to the first-stage data.

Let $U_{\alpha^{i}}(\alpha, \gamma)=\partial \log \operatorname{Pr}\left(D_{i} \mid G_{i}, E_{i} ; \alpha, \gamma\right) / \partial \alpha$ and

$U_{\gamma, i}(\alpha, \gamma)=\partial \log \operatorname{Pr}\left(D_{i} \mid G_{i}, E_{i} ; \alpha, \gamma\right) / \partial \gamma$ be the score functions;

$J_{\alpha \alpha}(\alpha, \gamma)=-\sum_{i \in \mathcal{S}_{1}} \partial U_{\alpha, i}(\alpha, \gamma) / \partial \alpha$,

$J_{\alpha \gamma}(\alpha, \gamma)=-\sum_{i \in \mathcal{S}_{1}} \partial U_{\alpha, i}(\alpha, \gamma) / \partial \gamma$ and $J_{\gamma \gamma}(\alpha, \gamma)=-\sum_{i \in \mathcal{S}_{1}} \partial U_{\gamma, i}(\alpha, \gamma) / \partial \gamma$ the submatrices of the information matrix for the first-stage case-control analysis based on the logistic regression model (1). At the second stage of the two-stage design proposed, a random subset of the first-stage cases is selected, and genotype data $\left\{G_{P i}, i \in \mathcal{S}_{F}\right\}$ are further collected for the parents of the selected second-stage cases, where $\mathcal{S}_{F}$ is the subset of $\mathcal{S}_{1}$ corresponding to the second-stage cases. Let $S_{i}(\beta)=\partial \log \operatorname{Pr}\left(G_{i} \mid G_{P i}, E_{i}, D_{i}=1 ; \beta\right) / \partial \beta$ and $I(\beta)=-\sum_{i \in \mathcal{S}_{F}} \partial S_{i}(\beta) / \partial \beta$ be the score function and information matrix for the CPG likelihood (3). Also, let $\mathcal{S}_{2}$ be the subset of $\mathcal{S}_{1}$ corresponding to the second-stage case-control sample, consisting of the second-stage cases (i.e. subjects in $\mathcal{S}_{F}$ ) and all the first-stage controls, and $K_{\alpha \alpha}(\alpha, \gamma)=-\sum_{i \in \mathcal{S}_{2}} \partial U_{\alpha, i}(\alpha, \gamma) / \partial \alpha, K_{\alpha \gamma}(\alpha, \gamma)=-\sum_{i \in \mathcal{S}_{2}} \partial U_{\alpha, i}(\alpha, \gamma) / \partial \gamma$, $K_{\gamma \gamma}(\alpha, \gamma)=-\sum_{i \in \mathcal{S}_{2}} \partial U_{\gamma, i}(\alpha, \gamma) / \partial \gamma$ the submatrices of the information matrix for the second-stage case-control analysis based on the logistic regression model (1).

Let $I=I(\widehat{\beta}), J_{*}=J_{\gamma \gamma}(\bar{\alpha}, \bar{\gamma})-J_{\alpha \gamma}(\bar{\alpha}, \bar{\gamma})^{T} J_{\alpha \alpha}^{-1}(\bar{\alpha}, \bar{\gamma}) J_{\alpha \gamma}(\bar{\alpha}, \bar{\gamma})$, and $K_{*}=K_{\gamma \gamma}(\widehat{\alpha}, \widehat{\gamma})-$ $K_{\alpha \gamma}(\widehat{\alpha}, \widehat{\gamma})^{T} K_{\alpha \alpha}^{-1}(\widehat{\alpha}, \widehat{\gamma}) K_{\alpha \gamma}(\widehat{\alpha}, \widehat{\gamma})$. Also, let $U_{*, i}(\alpha, \gamma)=U_{\gamma, i}(\alpha, \gamma)-J_{\alpha \gamma}^{T} J_{\alpha \alpha}^{-1} U_{\alpha, i}(\alpha, \gamma)$ and $Q_{*, i}(\alpha, \gamma)=U_{\gamma, i}(\alpha, \gamma)-K_{\alpha \gamma}^{T} K_{\alpha \alpha}^{-1} U_{\alpha, i}(\alpha, \gamma)$ be the efficient scores of $\gamma$ after accounting for the estimation of the intercept parameter $\alpha$ with the first and second-stage case-control samples. Recall that $\mathcal{S}_{1} \cap \mathcal{S}_{F}=\mathcal{S}_{F}$ and $\mathcal{S}_{2} \cap \mathcal{S}_{F}=\mathcal{S}_{F}$. Therefore the sandwich estimators for $\operatorname{Var}(\bar{\gamma})$, $\operatorname{Var}(\widehat{\gamma}), \operatorname{Cov}(\widehat{\gamma}, \bar{\gamma}), \operatorname{Cov}(\widehat{\beta}, \bar{\gamma})$ and $\operatorname{Cov}(\widehat{\beta}, \widehat{\gamma})$ are given respectively as

$$
\begin{aligned}
& \widehat{\operatorname{Var}}(\bar{\gamma}) \equiv V_{\bar{\gamma}}=J_{*}^{-1}\left\{\sum_{i \in \mathcal{S}_{1}} U_{*, i}(\bar{\alpha}, \bar{\gamma}) U_{*, i}^{T}(\bar{\alpha}, \bar{\gamma})\right\} J_{*}^{-1}, \\
& \widehat{\operatorname{Var}}(\widehat{\gamma}) \equiv V_{\widehat{\gamma}}=K_{*}^{-1}\left\{\sum_{i \in \mathcal{S}_{2}} Q_{*, i}(\widehat{\alpha}, \widehat{\gamma}) Q_{*, i}^{T}(\widehat{\alpha}, \widehat{\gamma})\right\} K_{*}^{-1}, \\
& \widehat{\operatorname{Cov}}(\widehat{\gamma}, \bar{\gamma}) \equiv C_{\widehat{\gamma}, \bar{\gamma}}=K_{*}^{-1}\left\{\sum_{i \in \mathcal{S}_{2}} Q_{*, i}(\widehat{\alpha}, \widehat{\gamma}) U_{*, i}^{T}(\bar{\alpha}, \bar{\gamma})\right\} J_{*}^{-1}, \\
& \widehat{\operatorname{Cov}}(\widehat{\beta}, \bar{\gamma}) \equiv C_{\widehat{\beta}, \bar{\gamma}}=I^{-1}\left\{\sum_{i \in \mathcal{S}_{F}} S_{i}(\widehat{\beta}) U_{*, i}^{T}(\bar{\alpha}, \bar{\gamma})\right\} J_{*}^{-1}, \\
& \widehat{\operatorname{Cov}}(\widehat{\beta}, \widehat{\gamma}) \equiv C_{\widehat{\beta}, \widehat{\gamma}}=I^{-1}\left\{\sum_{i \in \mathcal{S}_{F}} S_{i}(\widehat{\beta}) Q_{*, i}^{T}(\widehat{\alpha}, \widehat{\gamma})\right\} K_{*}^{-1} .
\end{aligned}
$$

The matrix $C(\beta, \gamma)=\operatorname{Cov}(\widehat{\beta}, \widehat{\gamma}-\bar{\gamma})$ can thus be estimated by $\left(C_{\widehat{\beta}, \hat{\gamma}}-C_{\widehat{\beta}, \bar{\gamma}}\right)$ and the matrix $V(\gamma)=\operatorname{Var}(\widehat{\gamma}-\bar{\gamma})$ can be estimated by $\left(V_{\widehat{\gamma}}+V_{\bar{\gamma}}-C_{\hat{\gamma}, \bar{\gamma}}-C_{\hat{\gamma}, \bar{\gamma}}^{T}\right)$.

When there are missing parents in the second-stage study, we propose using complete and incomplete trios and the conditional likelihood of Chen et al. [22] to obtain $\widehat{\beta}$. In this case the information matrix $I(\beta)$ and score function $S_{i}(\beta)$ for $\widehat{\beta}$ need to be modified to account for the nuisance parameters induced by missing parental data; please see the Appendix of Chen et al. [22] for expressions of the modified information matrix and score function for $\widehat{\beta}$. 


\section{References}

1 Devlin B, Roeder K: Genomic control for association studies. Biometrics 1999;55:9971004.

-2 Pritchard JK, Stephens M, Rosenberg NA, Donnelly P: Association mapping in structured populations. Am J Hum Genet 2000; 67:170-181.

>3 Satten GA, Flanders WD, Yang Q: Accounting for unmeasured population substructure in case-control studies of genetic association using a novel latent-class model. Am J Hum Genet 2001;68:466-477.

4 Zhu X, Zhang S, Zhao H, Cooper RS: Association mapping, using a mixture model for complex traits. Genet Epidemiol 2002;23: 181-196.

$\checkmark 5$ Price AL, Patterson NJ, Plenge RM, Weinblatt ME, Shadick NA, Reich D: Principal components analysis corrects for stratification in genome-wide association studies. Nat Genet 2006;38:904-909.

6 Kimmel G, Jordan MI, Halperin E, Shamir R, Karp RM: A randomization test for controlling population stratification in wholegenome association studies. Am J Hum Genet 2007;81:895-905.

7 Zhang F, Wang Y, Deng HW: Comparison of population-based association study methods correcting for population stratification. PLoS ONE 2008;3:e3392.

$\checkmark 8$ Marchini J, Cardon LR, Phillips MS, Donnelly P: The effects of human population structure on large genetic association studies. Nat Genet 2004a;36:512-517.

-9 Marchini J, Cardon LR, Phillips MS, Donnelly P: Genomic control to the extreme. Nat Genet 2004b;36:1129-1131.

-10 Spielman RS, McGinnis RE, Ewens WJ: Transmission test for linkage disequilibrium: the insulin gene region and insulin-dependent diabetes mellitus (IDDM). Am J Hum Genet 1993;52:506-516.

-11 Schaid DJ, Sommer SS: Genotype relative risks: methods for design and analysis of candidate-gene association studies. Am J Hum Genet 1993;53:1114-1126.
12 Curtis D: Use of siblings as controls in casecontrol association studies. Ann Hum Genet 1997;61:319-333.

13 Spielman RS, Ewens WJ: A sibship test for linkage in the presence of association: The sib transmission/disequilibrium test. Am J Hum Genet 1998;62:450-458.

14 Laird NM, Lange C: Family-based designs in the age of large-scale gene-association studies. Nat Rev Genet 2006;7:385-394.

15 Nagelkerke NJ, Hoebee B, Teunis P, Kimman TG: Combining the transmission disequilibrium test and case-control methodology using generalized logistic regression. Eur J Hum Genet 2004;12:964-970.

-16 Epstein MP, Veal CD, Trembath RC, Barker JN, Li C, Satten GA: Genetic association analysis using data from triads and unrelated subjects. Am J Hum Genet 2005;76:592608.

17 Chen YH, Lin HW: Simple association analysis combining data from trios/sibships and unrelated controls. Genetic Epidemiol 2008; 32:520-527.

18 Prentice RL, Pyke R: Logistic disease incidence models and case-control studies. Biometrika 1979;66:403-411.

19 Thomas DC: Case-parents design for geneenvironment interaction by Schaid. Genet Epidemiol 2000;19:461-463.

20 Chatterjee N, Kalaylioglu Z, Carroll RJ: Exploiting gene-environment independence in family-based case-control studies: increased power for detecting associations, interactions and joint effects. Genet Epidemiol 2005;28:138-156.

21 Chen YH, Chen H: A unified approach to regression analysis under double-sampling designs. J R Statistic Soc B 2000;62:449-460.

22 Chen YH, Lin HW, Liu H: Two-stage analysis for gene-environment interaction utilizing both case-only and family-based analysis. Genet Epidemiol 2009;33:95-104.
23 Allen AS, Rathouz PJ, Satten GA: Informative missingness in genetic association studies: case-parent designs. Am J Hum Genet 2003;72:671-680

24 Chen YH: New Approach to association testing in case-parent designs under informative parental missingness. Genetic Epidemiol 2004;27:131-140.

25 Little RJA, Rubin DB: Statistical Analysis with Missing Data. Hoboken, NJ, Wiley 2002 , ed 2.

26 Weinberg CR: Allowing for missing parents in genetic studies of case-parent triads. Am J Hum Genet 1999;64:1186-1193.

$>27$ Clayton DG: A generalization of the transmission/disequilibrium test for uncertainhaplotype transmission. Am J Hum Genet 1999;65:1170-1177.

28 Sun F, Flanders WD, Yang Q, Khoury MJ: Transmission disequilibrium test (TDT) when only one parent is available: the 1-TDT. Am J Epidemiol 1999;150:97-104.

$>29$ Cheng KF, Lin WJ: Simultaneously correcting for population stratification and for genotyping error in case-control association studies. Am J Hum Genet 2007;81:726-743.

>30 Kraft P, Yen YC, Stram DO, Morrison J, Gauderman WJ: Exploiting gene-environment interaction to detect genetic associations. Hum Hered 2007;63:111-119.

31 Teng J, Risch N: The relative power of familybased and case-control designs for linkage disequilibrium studies of complex human diseases. II. Individual genotyping. Genome Res 1999;9:234-241.

-32 McCarthy MI, Abecasis GR, Cardon LR, Goldstein DB, Little J, Ioannidis JPA, Hirschhorn JN: Genome-wide association studies for complex traits: consensus, uncertainty and challenges. Nat Rev Genet 2008;9:356-369.

33 Miclaus K, Wolfinger R, Czika W: SNP selection and multidimensional scaling to quantify population structure. Genet Epidemiol 2009;33:488-496. 